\title{
Cerebral cavernomatosis
}

\author{
Panos Ioannidis*, Dimitris Parissis, Theodora Afrantou and Georgios Psimmenos \\ Department of Neurology, AHEPA Hospital, Aristotle University of Thessaloniki, Kiriakidi 1,Thessaloniki, Greece
}

A 65 years old male was referred to our clinic for evaluation of persistent and diffuse headache the last 2 months. Neurological examination was normal and his past medical history was unremarkable. A brain MRI revealed multiple cerebral lesions (Figures 1a and 1b) compatible with cavernomas.

Cerebral cavernous malformation (CCM) are collections of small blood vessels in the brain and spinal cord with irregular structures such as thin walls, lack of supporting tissues with elastic fibers.

CCM are usually located in the white matter of the brain and spinal cord and present dynamic structures which are changing in size and number over time.

They can occur in sporadic and familiar forms. Sporadic forms present $80 \%$ of all cases. Typically, they are single lesions ( $40 \%$ of solitary cavernous malformations are correlated with venous malformations). At least $20 \%$, have the familiar form which is associated with multiplicity of lesions and propensity for growth.

All patients, especially these with multiple lesions should be tested for mutations in three genes (CCM1, CCM2, CCM3). Unfortunately, in our case the patient denied genetic testing. The exactly role of these genes remains unclear.

The most common symptoms are headache, epileptic seizures, memory-attention problems, visual disturbances and other deficits correlated with the area of the brain or spinal cord that is being involved. It is worth it to mention that a cavernous malformation could be present without any symptoms (12\%).

Brain MRI with gradient echo sequences are the most sensitive means for diagnosing CCM. Angiography is of a little value, because blood flows through these types of lesions slowly $[1,2]$.

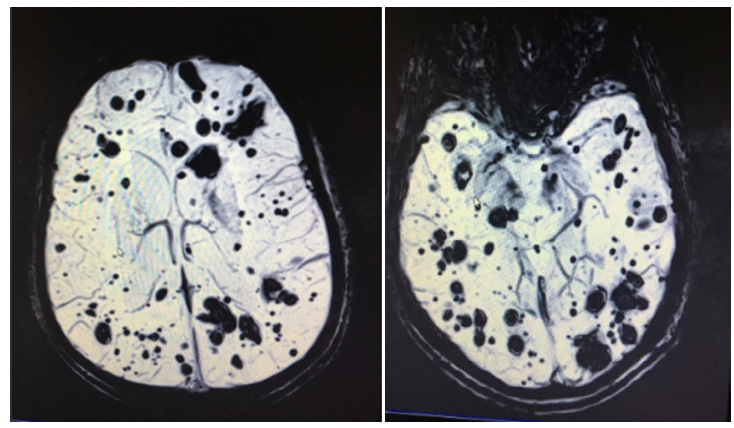

Figure 1a. Brain mri (Axial/swi images/): The MRI demonstrates multiple lesions ranging in size from microscopic to a number of millimetres in diameter.

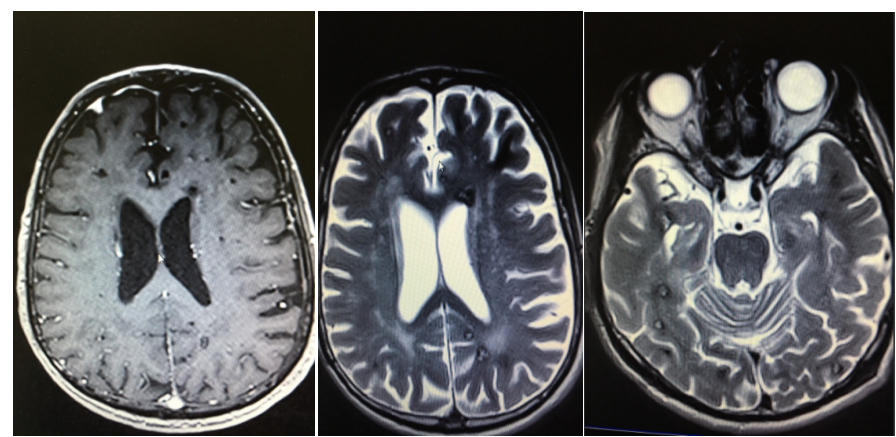

Figure 1b. Brain mri (Axial T1w and T2w images): The MRI demonstrates multiple lesions ranging in size from microscopic to a number of millimetres in diameter.

References

1. Mespreuve M, Vanhoenacker F, Lemmerling M (2016) Familial multiple cavernous malformation syndrome: MR features in this uncommon but silent threat. $J$ Belgian Soc Radiol 100: 51.

2. http://www.medscape.com/viewarticle/755276
Copyright: (C2017 Ioannidis P. This is an open-access article distributed under the terms of the Creative Commons Attribution License, which permits unrestricted use, distribution, and reproduction in any medium, provided the original author and source are credited.
Correspondence to: Panos Ioannidis, Department of Neurology, AHEPA Hospital, Aristotle University of Thessaloniki, Kiriakidi 1,Thessaloniki, Greece, Tel: (2310)993247; Fax: 2310994689; E-mail: ispanagi@auth.gr

Received: June 22, 2017; Accepted: July 08, 2017; Published: July 10, 2017 